\title{
The goal is to improve continuously
}

Since we assumed the editorship of the Revista Brasileira de Cirurgia Plástica/Brazilian Journal of Plastic Surgery (RBCP), we have strived to increase its quality in every way, using other journals in this field as our standard, according to their knowledge level. Despite all the care taken and opportunities to reduce errors, the inconveniences and omissions appear endless. Just when we believe we had resolved a particular issue, something completely unexpected comes up.

One such detail is the flaw in literature reviews. We have spoken, written, and published editorials, and have regularly sent messages to authors and even reviewers of the RBCP asking them to be accurate on this point. Any author would be honored on reading his or her name in the "literature cited" section of an article; the opposite is also true. Recently, fellow author Jaime Anger commented on the article "Deep venous thrombosis prophylaxis protocols comparative study: a new proposal" (Rev Bras Cir Plást. 2010;25(3):415-22), by Moulim et al. On that occasion, our fellow author argued that his name was not on the list of literature references even though his article on the subject was published in our journal. The situation prompted a comment that was published in the Letters to the Editor section with an appropriate apology. The problem was repeated when the article, "Surgical approach to the treatment of gynecomastia according to its classification" (Rev Bras Cir Plást. 2012;27(2):277-82), by Maia de Medeiros, did not cite the technique previously described and published by José Humberto Cardoso Rezende. The author of the article apologized for the omission in a letter sent to the editors. Based on ethical principles and not out of compulsion, the editors of the journal published the apology to Rezende, despite a lack of guilt and without regard to the motive of the complaint. For the sake of truth and formality, every author signs a statement, as described in the Instructions to Authors when submitting their article for publication, in which they assume full responsibility for the contents of the article. Since we assumed the editorship of the journal 4 years ago, this is the second time that comments of such nature have become public - we generally consider these to be of little concern. In truth, such omissions are frequent but are not rectified on the part of the non-cited authors.

Accurate literature reviews are a priority as they summarize what is already known about the subject matter and what an author effectively contributes to the existing body of knowledge, and confirms or fails to confirm its validity.

The Instructions to Authors included in all editions of our journal declare that an article can only be accepted when the candidate for publication signs the statement transferring copyright to the RBCP and assumes complete responsibility for the article's contents. The reviewers' job is to meticulously evaluate the articles, which are distributed according to the reviewers' previously registered fields of expertise. On that particular note, although Rezende be frustrated with the problem, he was right to charge the Editorial Board to be even more rigid than before. Requests for accountability of any sort strengthen quality improvement measures in all areas of human activity.

In fact, we have been sending circulars to reviewers requesting even more accurate assessments, although the responses have been insufficient. A new procedure of review control that will follow the articles sent to the reviewers is on track. The reviewers will have to answer each transcribed query on a printed sheet, which must accompany the reviewed article for quality control purposes.

Your suggestions will always be welcome.

The goal is to improve continuously.

\section{Ricardo Baroudi Editor}

\author{
Dov Charles Goldenberg \\ Associate Editor
}

